\title{
a feasibility study
}

George Kleftouris ${ }^{1}$, Elizabeth MA Hensor ${ }^{2}$, Paul J Harwood ${ }^{4}$, Nikolaos K Kanakaris ${ }^{4}$, Theodoros H. Tosounidis 3 , Peter Giannoudis ${ }^{4}$

${ }^{1}$ Leeds Teaching Hospitals NHS Trust, Leeds General Infirmary, Clarendon Wing Level A, Great George Street, LS1 3EX Leeds, UK, ${ }^{2}$ Leeds Institute of Rheumatic and Musculoskeletal Medicine, University of Leeds, NIHR-Leeds Biomedical Research Centre, Leeds Teaching Hospitals NHS Trust, Chapel Allerton Hospital, Leeds, ${ }^{3}$ University Hospital of Heraklion, Crete, Greece, ${ }^{4}$ Academic Department of Trauma \& Orthopaedics, School of Medicine, University of Leeds and NIHR Leeds Biomedical Research Unit, Chapel Allerton Hospital, Leeds, UK

\section{Purpose}

- One in three patients with hip fracture has a degree of cognitive impairment.

- A recent systematic report has shown that patients with cognitive impairment are rarely included in randomised control trials $(\mathrm{RCT})^{1}$.

- The purpose of this study was to evaluate the feasibility of performing an RCT including patients with and without dementia. In particular, we wanted to assess:

1. Whether we can recruit and retain patients with dementia in the study.

2. Whether 'Timed up and Go' (TUG) test can be used as the primary efficacy measurement tool in a full scale study.

\section{Methods}

- Inclusion criteria:

- Age 55-95 years old.

- Low energy OTA/AO type 31-A2 fractures.

- Patients able to walk $6 \mathrm{~m}$ prior to injury.

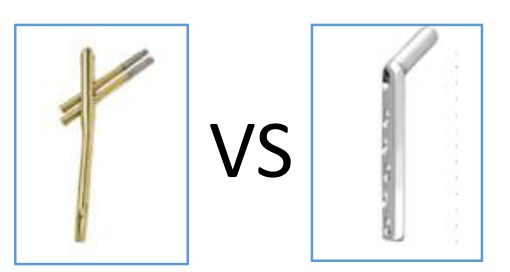

- Exclusion criteria:

- Pathological fractures.

- Other concomitant lower limb fractures.

- Survival <3 months or unfit to complete follow-up.

- Randomisation

- Online randomisation system.

- Stratified by Abbreviated Mental Test Score (AMTS).

- Permuted block randomisation.

- Consent:

- Written formal consent

- Personal consultee.

- Nominated consultee.

- Primary outcome: feasibility to perform trial including patients with AMTS $<8$.

- Secondary outcomes: perioperative parameters, functional outcomes.

- Follow-up in 2, 4 and 12 weeks.

- Financial support by Citieffe (Italy).

- Single centre trial.

- Local ethical committee approval.

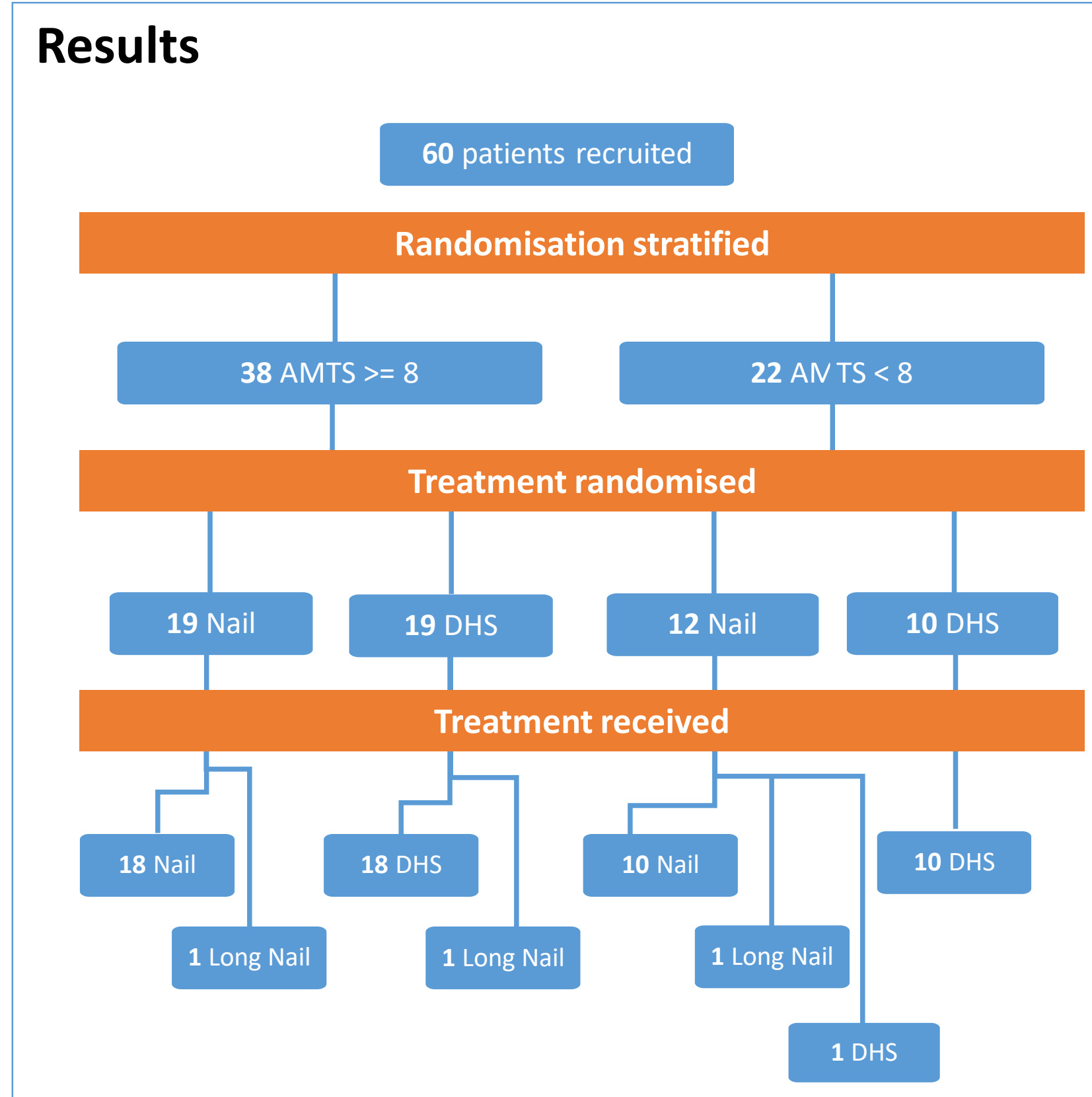

Diagram of patients' randomizations and treatment received stratified by AMTS score. Three patients received a long nail due to subtrochanteric extension of the fracture which was not obvious on plain radiographs. One patient received a DHS despite being randomised to the Nail group.

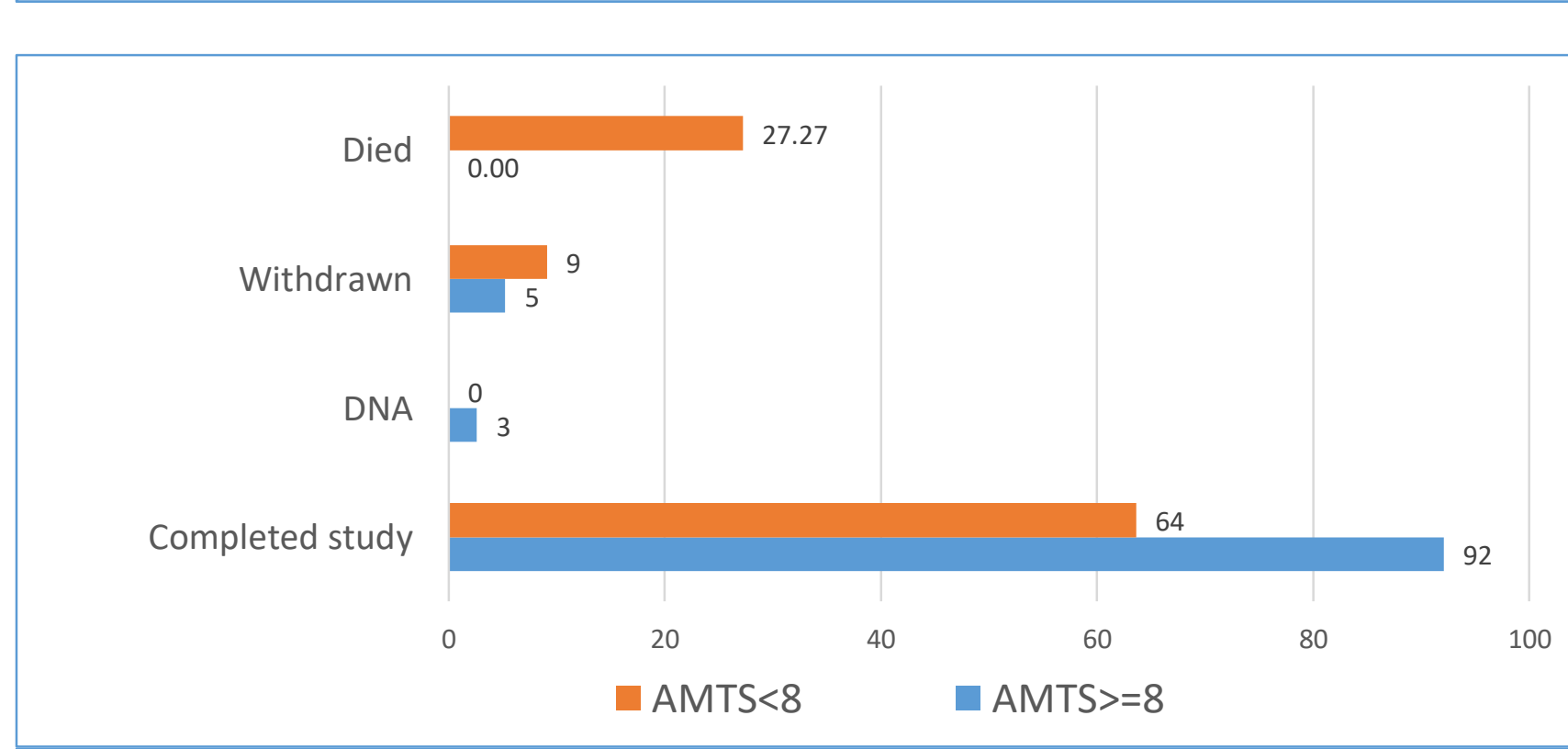

Proportion of patients completed the study and the reasons for dropouts.

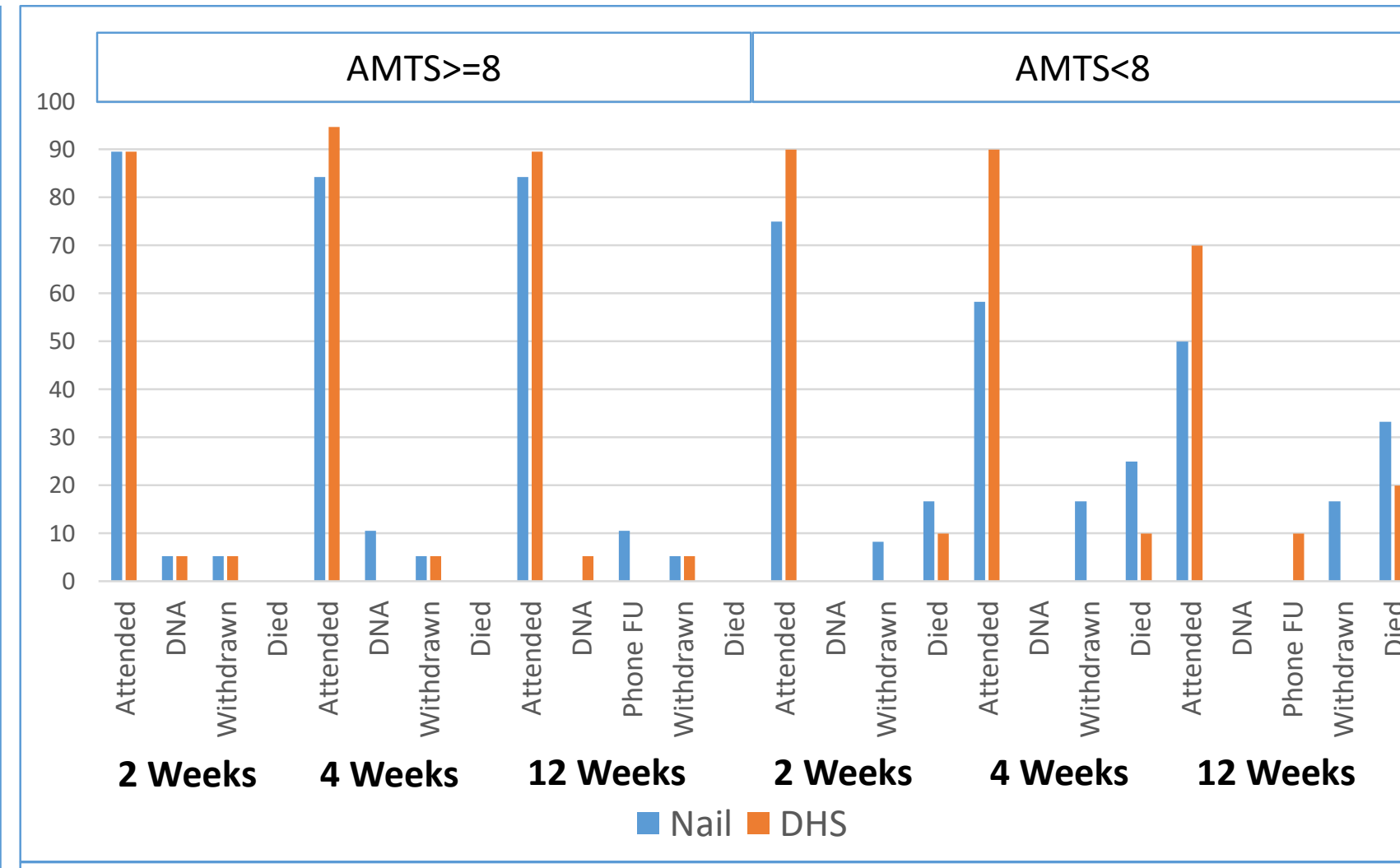

Proportion of patients attended each follow-up visit. Three patients were withdrawn because they received a long nail, 1 patients was withdrawn by their consultee, 6 patients with AMTS $<8$ died throughout the study, 2 patients with AMTS $>=8$ and 1 patient with AMTS $<8$ had a telephone FU in the last visit.

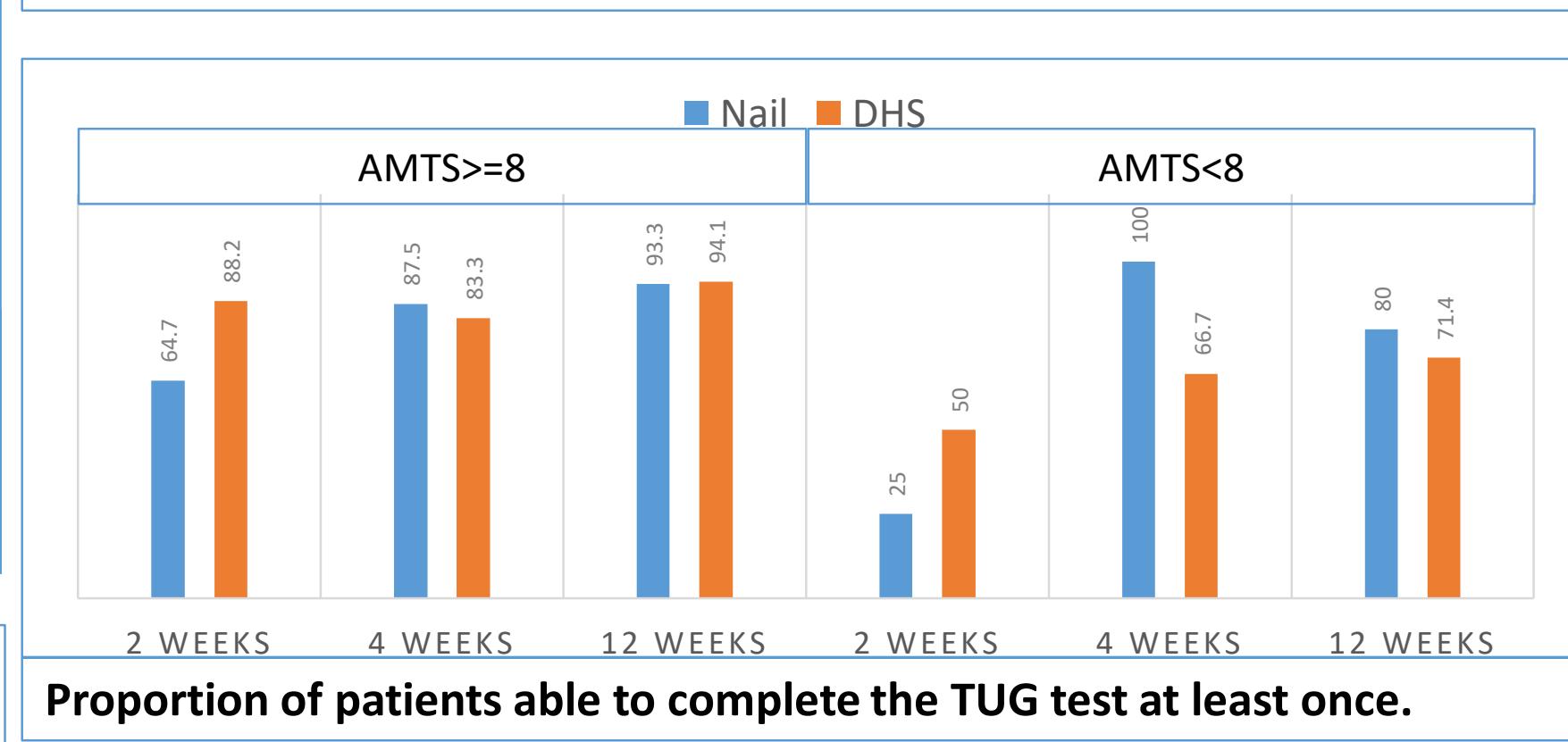

Proportion of patients able to complete the TUG test at least once.

\section{Conclusions}

1. Patients with AMTS $<8$ were less likely to complete the study.

2. Patients with AMTS $<8$ were less likely to be able to perform the TUG test at least once at each time point.

3. Patients with AMTS $<8$ had higher rates of drop-outs in each follow-up visit.

4. Overall, patients with AMTS $<8$ require different efficacy assessment tools than patients with intact cognition.

5. When designing a full scale trial, high number of drop outs are to be expected from AMTS $<8$ group. 